\title{
Visual perception of direction when voluntary saccades occur: \\ II. Relation of visual direction of a fixation target extinguished before a saccade to a subsequent test flash presented before the saccade ${ }^{1}$
}

\author{
LEONARD MATIN, ${ }^{2}$ ETHEL MATIN, AND JORDAN POLA \\ COLUMBIA UNIVERSITY
}

\begin{abstract}
Previously reported experiments demonstrated changes in the relation of visual direction to retinal locus for stimulation during voluntary saccades as compared to this relation before saccade initiation. The quantitative features of these results led to the prediction, confirmed in the present experiments, that there are shifts in visual direction for stimulation presented before the saccade itself. In the present report, monotonically increasing shifts were mapped with stimuli presented as early as 240 msec before the saccade up to the saccade itself. Such shifts cannot be accounted for readily by "inflowing" processes, and while "outflowing" processes seem to be implicated, their quantitative characteristics would need to be considerably different from those required by classical outflow theories.
\end{abstract}

The experiment to be reported here is one of a series directed at delineating mechanisms responsible for the normal stability of visual direction of stationary objects when the direction of gaze is changed. Theoretical interpretations of this phenomenon have posited the presence of an extraretinal signal ${ }^{3}$ regarding eye movements whose presumed influence is to modify the relation of visual direction to retinal locus so as to nullify (or cancel) the effect on visual direction of the eye-movement-produced retinal image shift. Our previous experiments (Matin \& Pearce, 1965; Matin et al, 1968; Matin, 1968; Matin, Matin, \& Pearce, 1969) have indeed confirmed the existence of an extraretinal signal related to voluntary saccades. However, some of the properties of this signal do not appear to conform to the main properties predicted by the classical theoretical treatments (Helmholtz, 1866; Sherrington, 1918) or by the Von Holst (1954) version of Helmholtzian outflow theory. In these experiments, the visual direction of a brief test flash presented when the eye was at a known point during a voluntary saccade (trigger point) was compared by the $S$ with the visual direction of a fixation target viewed and extinguished before the saccade. PSEs (points of subjective equality) for the fixation target shifted systematically in the direction of the saccade with increase in distance of the trigger point from the fixation target, instead of remaining fixed at the fixation target as would be predicted from the above-mentioned theories; furthermore, the shift was such as to indicate that the extraretinal signal was growing much more slowly than would be the case if this signal were to vary systematically with eye position as is predicted by these theories. Instead, the predominant features of the experiments so far reported strongly suggest that variation of extraretinal signal magnitude is time-locked to the saccade. While evidence for an effect of eye position on extraretinal signal magnitude has also been uncovered (Matin et al, 1968; Matin, 1968; Matin, Matin, \& Pearce, 1969; Matin et al ${ }^{4}$ ), its basis and characteristics are not clear, and some work now under way by one of us (JP) should clarify the issue considerably. In addition, it was pointed out (Matin, Matin, \& Pearce, 1969) that while the classical theoretical treatments would require that the extraretinal signal influence the visual direction of the target presented during the saccade, an equally plausible interpretation suggests that this signal influences the memory of visual direction of the view seen before the saccade had begun.

The observation that the extraretinal signal changes only slowly for flashes delivered at various trigger points during the saccade led us to the hypothesis that the signal grows over a period that is very much longer than the saccade itself. Further, early in the saccade, the magnitude of the extraretinal signal is sizable enough to suggest that the compensatory process would affect the reports if flashes were delivered prior to saccade onset. In the present experiments, we have confirmed the existence of such an anticipatory effect by investigating the visual direction of flashes delivered after a $S$ has been given a signal to turn his eye but before the eye movement begins.

\section{METHOD \\ Experimental Procedure}

The two Ss, EM and JP, had had considerable prior experience as $\mathrm{Ss}$ in similar experiments involving similar psychophysical discriminations. In those experiments, as in the present one, a tightly fitted scleral contact lens was worn on the left eye for use in the system measuring horizontal eye position. The S sat at a table in a completely dark room, with head position determined by a fitted mouth bite. Viewing was with the left eye; the right eye was occluded by an eye patch.

On each trial, the $S$ viewed a fixation target presented for $4 \mathrm{sec} ; 300 \mathrm{msec}$ after its termination, a 70-msec flash (first flash) occurred (see Fig. 1a for stimulus

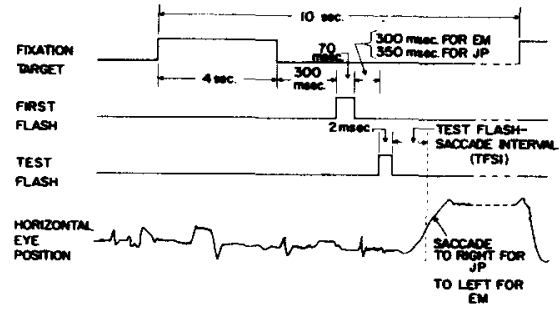

(a)

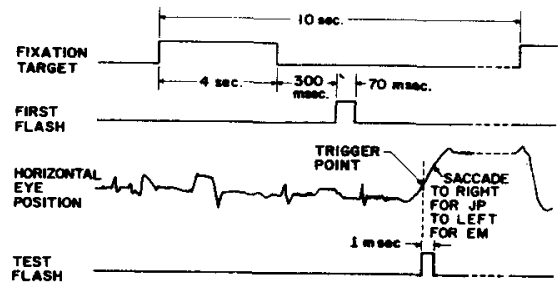

(b)

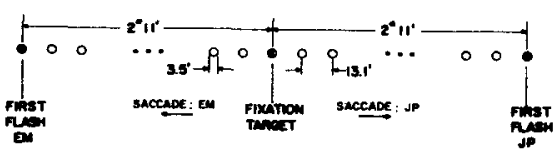

(c)

Fig. 1. (a) Temporal sequence of stimuli on trials in which the test flash was presented before the beginning of the saccade. (b) Temporal sequence of stimuli on trials in which the test flash was triggered by the eye movement. (c) Spatial array of stimuli; any target in the array could be used as a test flash. 
sequence). The first flash was presented $2 \mathrm{deg} 11 \mathrm{~min}$ to the right of the fixation target for JP and $2 \mathrm{deg} 11 \mathrm{~min}$ to the left of the fixation target for EM. Until the $S$ saw the first flash, he tried to keep his eye in the fixation position. When he saw the first flash, he attempted to saccade to the location at which it had been perceived and to remain in this new position until the next trial was initiated by the onset of the fixation target. (Due to the relative length of the first flash and reaction time, the flash was, of course, always over before the saccade began.) The $S$ was instructed to attempt to maintain a uniform reaction time in response to the first flash rather than to respond as quickly as possible. At a fixed duration after the onset of the first flash, a 2-msec test flash was delivered. The location of this flash was judged (with a report of left or right) relative to the location of the previously viewed fixation target. One of three test targets, each chosen on the basis of preliminary experiments to be within the S's uncertainty range, was presented per trial; the differently located test targets were ordered among trials in randomized blocks of three. Time between the onsets of first and test flashes was $300 \mathrm{msec}$ for EM and $350 \mathrm{msec}$ for JP. The choice of these durations was based on preliminary studies of reaction time (time from the end of the first flash to the moment when the eye crossed a predesignated point during the saccade) $; 5$ the values used were selected to yield a high proportion of trials on which the saccade would occur less than $100 \mathrm{msec}$ after the test flash and to minimize the number of trials on which the saccade began before delivery of the test flash. On each trial, the time from the end of the test flash to the moment when the eye crossed the predesignated position during the saccade (test flash-saccade interval, TFSI) was measured with a Beckman counter-timer, No.6144. The voltage, proportional to the average horizontal eye position during the presentation of the test flash, was measured with a second Beckman counter-timer, No.6144 (containing a No. 651 digital voltmeter plug-in module), that was gated by the timers controlling the onset and offset of the test flash. 6 With the information on eye position, values of retinal signal (i.e., horizontal distance of test flash from fixation target on the retina) were computed for each trial for the particular test flash used on that trial.

EM was run in this manner for 8 days, 60 trials per day. In order to facilitate a comparison of data collected under these conditions with those from experiments reported earlier (Matin, Matin, \& Pearce, 1969), in which the test flash was presented during the saccade, an additional 30 trials were run daily in which the test flash was triggered by an electrical signal taken from the system monitoring eye position when the eye reached a predesignated point in the saccade, the trigger point, $52.4 \mathrm{~min}$ to the left of the fixation position (Fig. 1b). The same point was used to measure the TFSI on trials with a fixed duration between the first flash and the test flash; since the test flash was delivered at the moment the fovea was at the trigger point, no further measure of eye position was required to define the retinal signal on these trials. The 30 triggered trials were run as a separate unit (Trials $1-30,31-60$, or 61-90), whose position within the daily total of 90 trials was chosen each day on a random basis. Three test targets, chosen to be in the uncertainty range for the triggered condition, were alternated in random blocks of three on the triggered trials.

A similar procedure was used with $\mathbf{J P}$, who was run for 7 days with 96 trials per day. In 72 of these trials, there was a predetermined time interval (350 $\mathrm{msec}$ ) between the first and test flashes; in the remaining trials, the test flash was triggered as the eye crossed a point $39.3 \mathrm{~min}$ to the right of the fixation target.

\section{Stimulus Array}

The stimulus array used for presenting the fixation target, first flash, and test flashes consisted of a horizontal series of circular targets, $3.5 \mathrm{~min}$ in diam at a viewing distance of $128 \mathrm{in.}$ (Fig. 1c). Adjacent targets were separated by $13.1 \mathrm{~min}$ of arc, and the array was distributed along a horizontal line that was perpendicular to the fixation axis when the $S$ viewed the member of the array used as the fixation target. Each target was constructed of an electronically controlled neon glow-discharge lamp, Dialite No. 38H, in front of which was a diffusing disk and a mask that provided a homogeneous circular disk of orange-red light (Matin \& Kibler, 1966). Ultraviolet radiation, invisible to the Ss, shone on the lamps continuously to provide stable flashing behavior (Matin, 1964b). All stimulus timing was performed by phantastron delay generators (reliability better than .5\%) that switched bistable multivibrators; the latter drove current generators providing the current to the glow-discharge lamps. Calibrations of stimulus duration were performed with the aid of a Beckman counter-timer, No. 6144.

\section{Measurement of Eye Position and Triggering of Test Flashes \\ Horizontal eye movements were monitored continuously during each experimental session by a contact lens}

technique described in detail elsewhere (Matin, 1964a; Matin \& Pearce, 1964; also see Figs. 3 and 4, Matin, "Matin, \& Pearce, 1969). The measuring system used an invisible (infrared) beam incident upon a plane circular mirror embedded in the temporal margin of a scleral contact lens that was worn on the S's left eye. As the eye rotated horizontally, more or less of the energy in the beam reflected from the contact-lens mirror passed a vertical straight edge and reached a photocell whose current output was linear with incident energy; the change in incident energy was linearly proportional to the angle of rotation. The output of this photocell was corrected for spurious variations through the use of a reference beam and a second photocell that monitored the total energy reflected from the contact-lens mirror. Since the field stop in the incident beam and its image at the straight edge were at optical infinity with respect to the contact-lens mirror, the system was insensitive to translations of the eye.

A corrected current from the first photocell was converted to a voltage and amplified. This voltage was then proportional to the horizontal angular position of the eye. On trials where the test flash was delivered at a fixed duration after the presentation of the first flash, this voltage was fed to the digital voltmeter, as described above.

On trials where the flash was delivered as the eye crossed the trigger point, the voltage proportional to eye position was adjusted prior to experimentation to be at zero with the eye fixating a steadily illuminated target at the trigger point. This zero was checked frequently during an experimental session and adjusted when necessary. The voltage measuring eye position was fed to a comparator whose output was a fixed positive voltage when the eye was on the fixation target side of the trigger point and a fixed negative voltage when the eye was on the other side of the trigger point. As the eye traversed the trigger point during a saccade, the comparator's output underwent a negative voltage step that was inverted and differentiated into a sharp positive pulse. This positive pulse started a delay generator set for $1 \mathrm{msec}$ and simultaneously turned on the test flash; the delay generator turned off the flash $1 \mathrm{msec}$ later. The delay between the eye's crossing of the trigger point and the turning on of the test flash was less than 3 microseconds. On trials where a fixed duration occurred between the first and test flashes, the output of the differentiator was also fed to the Beckman No.6144 counter-timer measuring TFSI, as described in the previous section. 
Table 1

Retinal and Target PSEs and JNDs as a Function of TFSI (Test Flash-Saceade Interval) for Subjects EM and IP. EM's saceades were to the left and JP's to the right. For trials on which teat flash presentation was trigered by the saccade the triger point was $52.4 \mathrm{~min}$ to the left of the fixation target for EM and $39.3 \mathrm{~min}$ to the right for JP. $L$ and $R$ refers to left and right respectively. $N$ is the number of trials.



The current-voltage transduction of the output of the photocells, amplification, comparison, integration, differentiation, and pulse shaping were performed by chopper-stabilized operational amplifiers (Philbrick SP656 or Sk2-V/Sk2-P). Continuous records of eye movements, stimulus sequence, and S's psychophysical response were recorded on a Honeywell Visicorder No. 1508.

\section{RESULTS}

The data from the trials on which there was a fixed duration between the first flash and test flash were processed as follows: Trials on which the flash occurred after the saccade had begun were discarded; the remaining data were grouped according to
TFSI, as shown in the first column in Table 1. ${ }^{7}$ For each TFSI group and for the data on the triggered trials, target PSEs (points of subjective equality) and JNDs (just noticeable differences) were calculated from the psychometric function relating the target location of the test flash to the probability of a report that the test flash appeared to the right of the fixation target. ${ }^{8}$ These target PSEs and JNDs are shown for JP and EM in Table 1; the PSEs are also graphed in Fig. 2. For JP, a sizable displacement of PSE from the fixation target was present at all values of TFSI; for EM, these displacements were much smaller. However, for both Ss, as TFSI decreased, the target PSE shifted further in the direction opposite to that of the

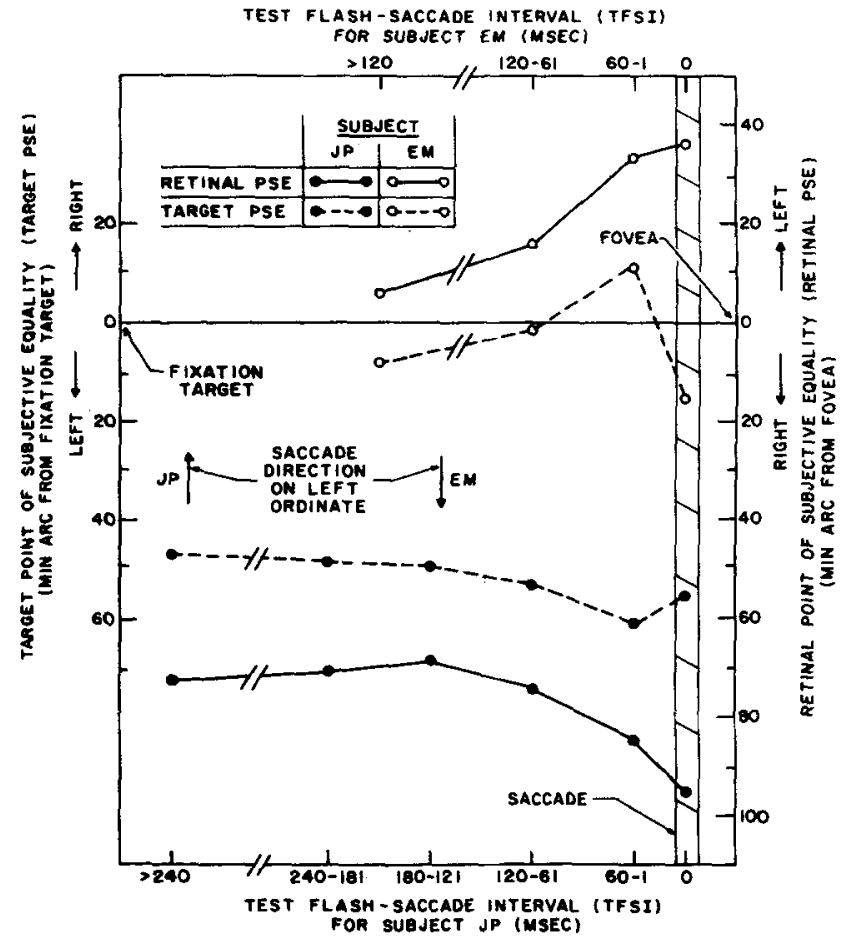

subsequent saccade. This systematic effect did not extend to the period during the saccade where, for both Ss, the target PSE shifted in the same direction as the saccade relative to where it had been in the immediately preceding interval.

This latter reversal $(27.5 \mathrm{~min}$ for $\mathrm{EM}$, $5.3 \mathrm{~min}$ for JP) for target PSEs is not present in the retinal PSEs ${ }^{9}$ (points of subjective equality on the retina) shown in Fig. 2 and Table 1. For both Ss, displacement of the retinal PSE from the fovea increased monotonically with decrease in TFSI; this monotonic shift continued into the period of the saccade itself. The displacement was consistently larger for JP than for EM at all values of TFSI. For EM, the retinal PSE for flashes presented $120 \mathrm{msec}$ or more prior to the onset of the saccade departed only slightly from zero $(5.8 \mathrm{~min}$ to the left of the central fovea). Her retinal PSE was $36.2 \mathrm{~min}$ to the left of the central fovea for flashes presented during the saccade and $33.6 \mathrm{~min}$ to the left of the fovea for flashes presented in the $60-\mathrm{msec}$ period prior to the saccade. For JP, retinal PSE was $1 \mathrm{deg} 35.2 \mathrm{~min}$ to the right of the fovea when the test flash was presented during the saccade and $10.3 \mathrm{~min}$ closer to the fovea during the $60-\mathrm{msec}$ period prior to the saccade; even for TFSIs between 181 and $240 \mathrm{msec}$, the retinal PSE was still $70.9 \mathrm{~min}$ from the fovea. Since the test flash preceded the saccade by more than 240 msec on only 35 trials and no trials occurred in which the TFSI was greater than $450 \mathrm{msec}$, it was not possible to determine how early the shift of PSE from the fovea began for this $S$. But the relatively large shift for TFSIs greater than 240 msec was consistent from day to day; it was also found when the $S$ was tested with a different time interval between the first flash and the test flash (100 msec) and with a different standard target (the first flash).

The difference between target and retinal PSE for a given TFSI is a reflection

Fig. 2. Points of subjective equality (PSEs) for the fixation target as measured by test flashes presented before and during the saccade. Target PSEs (dashed lines) are angular distances from the fixation target at the stimulus array (left ordinate). Retinal PSEs (solid lines) are angular distances from the fovea messured at the retina (right ordinate). For each $S$, the retinal PSE at a given test-flash/saccade interval (TFSI) is calculated from the same trials as the corresponding target PSE. Note that upward is rightward on the left ordinate and leftward on the right ordinate (reversal resulting from inversion of retinal image by ocular dioptrics). 


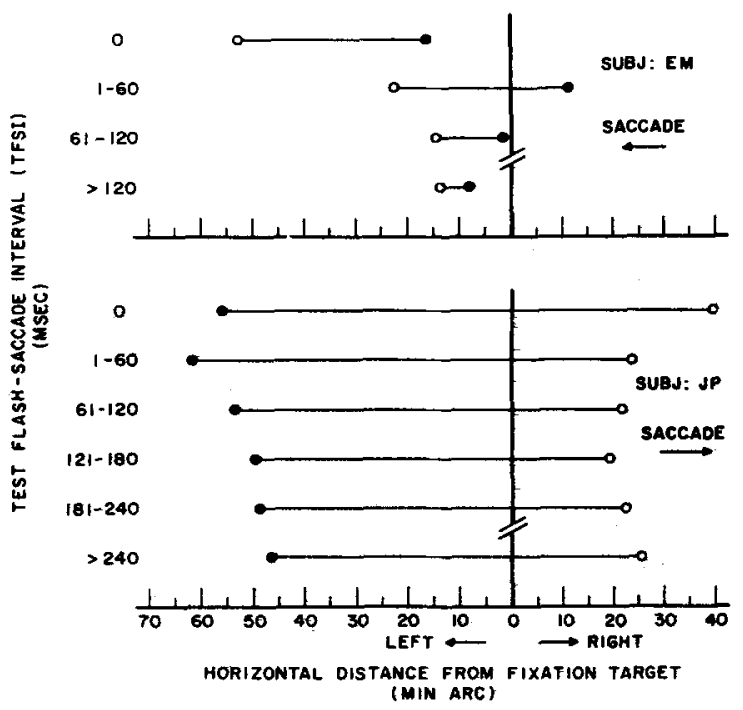

of the amount of eye movement (mostly drift) between fixation-target extinction and test-flash presentation, mentioned earlier in Note 5; this fact is shown more clearly in Fig. 3, which displays the target and retinal PSEs in such a way as to indicate also a measure of eye position at the moment of flash presentation. In Fig. 3, the spatial dimension is represented by the horizontal coordinate with location of the fixation target at the origin. The filled circle represents the location of the target PSE. The line connecting the open and filled circles at each TFSI represents the magnitude of the retinal PSE, and the open circle represents a measure of average eye position. Although this value for eye position is itself not a directly measured value (since it is the difference between the retinal and target PSEs that are themselves theoretical parameters of best-fitting ogives), it does represent accurately the fact that during the interval between fixation-target extinction and the saccade beginning the eye tends to drift in the direction of the subsequent saccade (further experiments designed to look at this fact directly will be presented in a subsequent report). Modal values of eye position at the time of test-flash presentation obtained directly from eye-position distributions that included all trials for which TFSI $>0$ was 12 min to the left of fixation position for EM and $18 \mathrm{~min}$ to the right of fixation position for JP.

These results, then, show that the failure of complete compensation for eye movements that was reported earlier in this series for flashes presented at a number of points during the saccade (and repeated here for one point) has its counterpart for flashes presented prior to the saccade. Here, shifts of PSE occur in a direction that is appropriate to compensate for the subsequent saccade as well as the preceding drift but are completely inappropriate in magnitude to be effective in maintaining the PSE at the fixation target.

\section{DISCUSSION}

\section{The Shifts are Saccade-Contingent}

The main concern of this report is with possible changes of visual direction for specific retinal loci in the period prior to the beginning of a voluntary saccade. The data are clear in demonstrating the presence of systematic changes in a direction that would be compensatory for the subsequent saccade, that these shifts can be quite sizable, can occur with stimuli presented as early as $240 \mathrm{msec}$ prior to the saccade, and that they increase monotonically as the moment of saccade occurrence is approached; in addition, the monotonic increase is continuous with the shift magnitude during the saccade. These characteristics strongly imply that these shifts are saccade-contingent.

Further support for this results from comparison of these shifts with shifts that occur in the dark when Ss attempt to hold their eyes steadily in a position of prior fixation: In the present experiment, the interval between extinction of the foveal fixation target and presentation of the test flash was $650 \mathrm{msec}$ for $\mathrm{JP}$ and $600 \mathrm{msec}$ for EM. JP's retinal PSEs were about 70-85 min from the fovea and EM's were 5-35 min, depending on TFSI. In other experiments (Matin, Matin, \& Pola, 1969), Ss reported on the horizontal vernier offset of two briefly flashed lines occurring with a short temporal interval between them; the retinal PSEs for the line flashed first (the S reported on the position of the second with regard to the first line) were 4 and $7 \mathrm{~min}$ for JP and 5 and $6 \mathrm{~min}$ for EM when
Fig. 3. Target PSEs in minutes of arc from the fixation target (filled circles) are shown for various values of test-flash/saccade interval (TFSI). The absolute value of the retinal PSE is shown on the same seale by the length of the line connecting filled and unfilled circles. The unfilled circle is thus a measure of central tendency for the eye position during the test flash (derived from the values for target and retinal PSEs).

the intervals between presentation of the two lines were 500 and $800 \mathrm{msec}$, respectively. Retinal JNDs in this latter study were all under $10 \mathrm{~min}$; retinal JNDs in the present study are all greater than $10 \mathrm{~min}$. Some portion of the differences between results in the two kinds of experiments may be due to the difference in stimulus characteristics. However, in a brief control experiment on JP, carried out in connection with the present study, stimuli were presented in the same sequence as in the main experiment described above, but no saccades were made; under these conditions, the shift in retinal PSE was about $1 / 10$ the size of the values shown for JP in Table 1, and thus similar to the results with the vernier targets when saccades also were not attempted.

The similarity of EM's retinal PSE for TFSI $>120 \mathrm{msec}$ to her retinal PSE for comparable interstimulus intervals in the vernier acuity study suggests that in the present experiments the shift for TFSI $>120 \mathrm{msec}$ is not necessarily saccade-contingent and could be related to events prior to planning of the saccade. On the other hand, the difference for JP between the retinal PSE at even the largest TFSI obtainable here and the retinal PSE for the identical stimulus sequence when no saccade was attempted, as well as similarity of the latter to the retinal PSE in the vernier acuity study, suggests that planning of the saccade was a much more lengthy process for him than it was for EM, and that it began sooner after the first flash or possibly even before it.

\section{An Inflowing Signal Would Be Too Late To Be Useful}

The presence of such sizable shifts for such large values of TFSI is extremely difficult to account for on the basis of inflow theory: With TFSI $=240 \mathrm{msec}$, the test flash will have been extinguished $240 \mathrm{msec}$ before the voluntary saccade began. During the TFSI, the eye continues to drift slowly as it has since the fixation target has been extinguished, typically with a preponderance of movement in the direction in which the saccade will occur; 
saccades in this interval are rare, and those that do occur are small enough to fall into the class that is generally labeled "involuntary." Eye movements between fixation-target extinction and the occurrence of the voluntary saccade thus could not be expected to give rise to inflowing signals of sufficient magnitude to result in the observed shift. 10 Further, although an overall change in tonic contraction level of the extrinsic eye musculature in the TFSI that did not result in eye-position change is possible, it would have to be essentially isometric and dynamically balanced between antagonistic muscles; and, although such a change could conceivably provide inflowing signals sufficiently early to be useful, it is extremely difficult to see how such a balanced set of signals could produce a directional bias of visual direction.

For values of TFSI equal to or larger than $240 \mathrm{msec}$, it is likely that the test flash was, in fact, seen before the voluntary saccade began; such an inference is reasonably made from classical reaction-time data. However, the $S$ 's directional report was a two-alternative forced choice for which reaction time is known to be typically longer than for the simple reaction time to the presence of a light, and it is thus possible that the directional judgment was formed during, or somewhat after, the saccade, even for the longer values of TFSI. Even so, it does not appear likely that an inflowing signal arising from muscular contractions during the saccade could have been registered sufficiently early to influence the report of direction. However, in this regard, it is worth noting that in another study (Matin et $\mathrm{a}^{4 b}$ ) we have found that introduction of a visual context as long as $150 \mathrm{msec}$ after presentation of a test flash in the middle of a voluntary saccade had a significant influence on the directional report of EM; JP's reports, however, were only slightly influenced, even when the context was introduced as little as $1 \mathrm{msec}$ after the test flash. The context in all cases must have been seen after the test flash was seen. Such evidence-that events occurring after the test flash is likely to be seen can influence the report of direction-leaves open the possibility that inflowing signals arising from the saccadic eye movement could influence the reports in the present experiments even for large values of TFSI. While the present data, particularly for JP, do weigh heavily against this possibility, they thus cannot by themselves be considered conclusive.

The previous discussion indicates that an inflowing signal consequent to the voluntary saccade would probably be too late to influence the directional report when test flashes are presented as much as
$240 \mathrm{msec}$ before the saccade; such an argument does not hold for an outflowing signal. Since the intention to turn the eye precedes the actual change in eye position, an outflowing extraretinal signal could arrive early enough to be of use in influencing the directional reports.

Classical Inflow and Outflow Theories Employ the Wrong Extraretinal Signal

Classical inflow and outflow theories were both essentially designed to account for the fact that stationary objects normally do not appear to move when eye movements shift the direction of gaze from one point to another in the visual field. Since the extraretinal signals required by these theories were designed to account for visual stability, their quantitative features cannot also account for cases of instability. It is clear that instability is the rule, however, in the present experiments. These experiments depart from conditions in a normal viewing situation in three ways: (1) A stable visual background is absent in the present experiments; (2) the standard target in the comparison-the fixation target-is removed before the saccade begins; and (3) the comparison target is presented as a flash. However, it should be noted that these differences do not violate the conditions required for stability under the classical theories and should thus be irrelevant to the production of the kind of extraretinal signal required by classical outflow or inflow theory and its consequent influence on the directions at which the visual targets involved in the comparison are seen. That is, the extraretinal signals assumed by the classical theories are tied to the intention regarding the eye movement or the eye movement itself; as such, they should not be influenced by the details of the visual stimulation per se.

The compensatory extraretinal signals of inflow and outflow theories must be equal in magnitude and opposite in direction to the shift of the image of a stationary target across the retina, and the growth of this signal must be parallel (with inverted sign) to the change of eye position during the saccade itself (see Fig. 9a, Matin, Matin, \& Pearce, 1969). The magnitude of such a signal is, of course, tied to the position of the eye. The classical theories require such a signal, and only such a signal, to account for visual stability when saccades occur. IAlthough outflow theory ties the extraretinal signal to the intention to turn the eye, the requirement that such a signal also be tied to eye position follows immediately if it is required that this signal account for visual stability; such a requirement is, in fact, manifest in the von Holst (1954) version (see Footnote 13, Matin, Matin, \& Pearce, 1969).] However, the slow change in PSE when flashes are presented in the period prior to the beginning of the saccade, as well as the very small further change when flashes are presented during the saccade, are incompatible with the presence of an extraretinal signal with these quantitative properties.

\section{Some Relations to Normal Viewing}

Since the PSEs obtained in the present experiments are not those we would expect if complete and accurate compensation for change in eye position occurred, we might expect to find shifts in visual direction or perhaps apparent movement when voluntary saccades occur in normal environments. Subjective reports of movement are not common, however, when the visual field is stationary. At least one reason must be related to the difference in the temporal aspects of the stimulation between our present experiments and stimulation provided from stationary continuously present objects. Thus, in our experiments, at the moment that the test flash is seen, the fixation target is available only in memory and has been in memory for a duration that is roughly equal to the period between fixation target extinction and test flash presentation (600 and $650 \mathrm{msec}$ for JP and $E M$, respectively, on trials in which the test flash is presented before the saccade, somewhat longer durations on trials in which the test flash is presented during the saccade). In normal environments, an object that is fixated before a saccade remains available as a stationary visual stimulus during this interval; thus, any memory of fixation-target location during the presaccadic period corresponding to the interval between fixation-target extinction and test flash presentation may be continuously updated by new perceptual information. However, in experiments in which a comparison target directly above the fixation target remained on during the entire presaccadic interval as well as during the saccade, PSEs measured by test flashes during the saccade are shifted inappropriately for accurate compensation (Matin, Matin, Pola, \& Kowal, 1969). Thus, the occurrence of shifts in PSE measured by test flashes prior to the saccade may not be a simple consequence of early fixation target extinction.

In work preliminary to the experiments in which the comparison target lay above the fixation target and remained on during the entire presaccadic interval and the saccade, the fixation target itself was left on during this period. While the eye carried out the saccade, the region of retina stimulated by the test flash received stimulation from the fixation target; the test flash itself then could not be seen. Such masking by subsequent stimulation is 
potentially an ever-present concomitant of eye movements in normally lit environments. Depression of visual sensitivity in the presaccadic interval (Volkmann, 1962; Latour, 1962; Zuber \& Stark, 1966; Volkmann et al, 1968) might st ?plement such an effect. This suggests that some essential factor in the appearance of stability for stationary objects present during the presaccadic period resides in the continuous presence of such objects, and that our ability to measure shifts in the present type of experiment depends on our utilization of flashed stimulation. ${ }^{11}$

\section{REFERENCES}

FINNEY, D. J. Probit analysis Cambridge, England: Cambridge University Press, 1947.

HELMHOLTZ, H. von. Handbuch der Physiologischen Optik. Leipzig: Voss, 1866. English translation from Ed. 3, 1925, J. P. C. Southall (Ed.), A treatise on physiological optics Vol. 3. New York: Dover, 1963.

HOLST, E. von. Relations between the central nervous system and the peripheral organs. British Journal of Animal Behaviour, 1954, 2, 89-94.

LATOUR, P. L. Visual threshold during eye movements. Vision Research, 1962, 2, 261-262.

Matin, E., MATIN, L., POLA, J., \& KOWAL, $K$. The intermittent light illusion and constancy of visual direction during voluntary saccades. Paper presented at the meeting of the Psychonomic Society, November 1969.

MATIN, L. Measurement of eye movements by contact-lens techniques: Analysis of measuring systems and some new methodology for three dimensional recording. Journal of the Optical Society of America, 1964a, 54, 1008-1018.

MATIN, L. Use of the glow modulator tube for visual research. American Journal of Psychology, 1964b, 77, 650-651.

MATIN, L. Eye movements and visual direction. Sy m o sium: Problems of sensory-motor-control in the C.N.S.: The role of feedback, feed forward and the corollary motor discharge. American Psychological Association, September 1968.

MATIN, L., \& KIBLER, G. Acuity of visual direction in the dark for various positions of the eye in the orbit. Perceptual \& Motor Skills, $1966,22,407-420$.

MATIN, L., MATIN, E., \& PEARCE, D. G. Visual perception of direction when voluntary saccades occur: I. Relation of visual direction of a fixation target extinguished before a saccade to a flash presented during the saccade. Perception \& Psychophysics, 1969, 5, 65-80.

MATIN, L., MATIN, E., \& POLA, J. Detection of vernier offset, eye movements, and autokinetic movement. Paper presented at the meeting of the Eastern Psychological Association, 1969.

MATIN, L., MATIN, E., POLA, J., \& PEARCE, D. G. Visual perception of direction for stimuli flashed before, during, and after voluntary saccadic eye movements. Paper presented at the meeting of the Psychonomic Society, October 1968.

MATIN, L., \& PEARCE, D. G. Three-dimensional recording of rotational eye movements by new contact-lens technique. In W. E. Murry and P. F. Salisbury (Eds.), Biomedical science instru:nentation. New York: Plenum Press, 1964. Pp. 79-95.

MATIN, L., \& PEARCE, D. G. Visual perception of direction for stimuli flashed during voluntary saccadic eye movements. Science, $1965,148,1485-1488$.

MATIN, L., PEARCE, D. G., MATIN, E., \& KIBLER, G. Visual perception of direction in the dark: Roles of local sign, eye movements and ocular proprioception. Vision Research, $1966,6,453-469$.

SHERRINGTON, C. S. Observations on the sensual role of the proprioceptive nerve-supply of the extrinsic ocular muscles. Brain, 1918 41, 323-343.

VOLKMANN, F. Vision during voluntary saccadic eye movements. Journal of the Optical Society of America, 1962, 52, 571-578.

VOLKMANN, F., SCHICK, A. M. L., \& RIGGS, L. A. Time course of visual inhibition during voluntary saccades. Journal of the Optical Society of America, 1968, 58, 562-569.

ZUBER, B. L., \& STARK, L. Saccadic suppression: Elevation of visual threshold associated with saccadic eye movements Experimental Neurology, 1966, 16, 65-79.

\section{NOTES}

1. This research was supported by Nationa Science Foundation Research Grants GB-4263 and GB-5947 and by PHS Research Grant NB-07547 from the National Institute of Neurological Diseases and Blindness, National Institutes of Health.

2. Address: Department of Psychology, Columbia University, New York, N.Y. 10027.

3. We shall use the term "extraretinal signal" to refer to any signal not derived from the retina regarding eye position, change in eye position, intention to move the eye, or shift in attention that might contribute to the report of relative visual direction.

4. (a) Matin, L., Matin, E., \& Pola, J. Visua perception of direction when voluntary saccades occur: III. Relation of visual direction of fixation target extinguished before a saccade to a test flash presented after the saccade. In preparation. (b) Matin, L., Matin, E., \& Pola, J. Visual perception of direction when voluntary saccades occur: IV. The influence of some temporal variations in context when a light flash is presented in the middle of a saccade. In preparation. (c) Matin, L., Matin, E., \& Pola, J. Detection of vernier offset for unequal luminance flashes presented during voluntary saccades. In preparation.

5. In measuring time of onset of the saccade, it is inconvenient to select the moment at which the eye crosses a point very close to the actual position of the eye at the beginning of the saccade since variation in eye position during the fixation period and during the period in the dark before the beginning of the saccade is such that the selected point would be crossed frequently before the saccade began. Accordingly, to define the time of occurrence of the saccade, the moment that the eye crossed a point $52.4 \mathrm{~min}$ to the left of the fixation position was chosen for EM and a point $39.3 \mathrm{~min}$ to the right of the fixation position for JP. The time from the actual beginning of the saccade to the time when the eye reached the predesignated position was less than $5 \mathrm{msec}$ for JP and less than $10 \mathrm{msec}$ for EM.

6 . Since the eye drifted in the darkness between removal of the fixation target and presentation of the test flash, accurate knowledge of the retinal area stimulated by the test flash requires a direct measurement of eye position when the flash occurs.

7. The data were grouped into intervals of $60 \mathrm{msec}$ along the TFSI dimension for calculation of PSEs after inspection of the graphical distributions. The graphs clearly indicated a monotonic shift of the retinal PSE with TFSI, but not enough data were available to warrant a finer grouping. In E.M.'s data, the 121. to 180 - and 181 - to $240-\mathrm{msec}$ intervals did not contain enough measurements to calculate separate PSEs for these intervals. The density of the measurements along the TFSI dimension was of course, not uniform over the range in which measurements were obtained; hence, the PSEs were calculated with unequal $\mathbf{n}$.

8. PSEs were the $50 \%$ points and JNDs, the standard deviations of the underlying densities corresponding to the best fitting normal ogives (maximum likelihood criterion). These calculations were made on the IBM 7094 computer at the Columbia Computing Center by Dr. Joyce Kerr, using the probit method (Finney, 1947).

9. The retinal PSE is the point of subjective equality for the standard target as measured by the test flashes and expressed in units of horizontal angular distance from the fovea.

10. If the target JNDs were smaller than the retinal JNDs for nonzero values of TFSI, it would be possible to infer that the directional reports reflect a process that is at least in part compensatory for the involuntary eye movement that occurs between fixation-target extinction and presaccadic test-flash presentation. Small bu consistent differences (seven out of eight possible comparisons) in this direction were in fact obtained (Table 1). Due to the necessity for grouping the data over sizable ranges of TFSI and retinal signal in order to calculate the retinal JNDs and over the same ranges of TFSI to calculate the target PSEs, however, the calculated values are somewhat inflated as result of grouping errors. It is not possible to calculate the amoun of inflation from these sources with any exactness. They are potentially sufficiently large so that it is not feasible to attempt to draw any conclusions here with regard to a compensatory process operating with reference to the involuntary eye movements that do occur between fixation-target extinction and presaccadic test-flash presentation. (It should be pointed out that grouping of data would not be expected to have any important biasing effects on retinal PSEs.)

11. It is worth noting that the magnitude of our measured shift is above the threshold for movement of a continuously present object during steady fixation (e.g., $60 \mathrm{~min} / 650 \mathrm{msec}$ for JP). It is possible that the threshold for movement rises in the presaccadic interval, but we know of no evidence to support this conjecture at present. 